\title{
Produção de Leite e Comportamento de Amamentação em Cinco Sistemas de Produção de Gado de Corte ${ }^{1}$
}

\author{
Ana Carolina Espasandin², Irineu Umberto Packer³, Maurício Mello de Alencar4
}

\begin{abstract}
RESUMO - Foram estudados a produção de leite de vacas Nelore e o comportamento de amamentação em diferentes sistemas de produção: NR-Nelore Referência, sob manejo extensivo (manejo tradicional); NI-Nelore, sob manejo intensivo; e três cruzamentos CNCanchim x Nelore, AN-Angus x Nelore e SN-Simental x Nelore, sob manejo intensivo. Em três momentos da lactação (60, 120 e 180 dias após o parto), foram medidos, nos bezerros, o número e a duração das mamadas, o ganho diário de peso (kg/dia) e o peso à desmama. O momento da lactação e a interação sistema de produção x momento da lactação apresentaram efeito significativo sobre a produção de leite. A produção de leite não apresentou corrrelação com o comportamento de amamentação nem com o ganho de peso dos bezerros dos diferentes sistemas de produção. Condições deficientes de alimentação não resultaram em menores produções de leite de vacas Nelore, mas sim em acentuadas perdas de peso $(80 \mathrm{~kg})$ durante a estação de monta no sistema NR. O tempo diário de amamentação apresentou diminuições significativas no sistema extensivo com o decorrer da lactação, enquanto os sistemas intensivos não mudaram ou aumentaram os minutos de amamentação por dia. Para as condições nas quais o experimento foi desenvolvido, os bezerros cruzados apresentaram os melhores desempenhos durante a fase pré-desmama, em comparação com os bezerros Nelore.
\end{abstract}

Palavras-chave: comportamento de amamentação, gado de corte, produção de leite

\section{Milk Yield and Suckling Behavior in Five Beef Cattle Production System}

\begin{abstract}
Milk yield in Nellore cows and suckling behavior of their calves of different production systems: NR- Extensive Nellore, NI- Intensive Nellore; and three crossbreeding systems (CN-Canchim-Nellore, AN-Angus-Nellore and SN-Simmental-Nellore in intensive management), were studied. Milk production of cows and number and length of suckles, and daily gain (kg/day) of calves were obtained in three moments of lactation (60,120 and 180 days after calving). Moment of lactation and production system by lactation moment interaction had a significant effect in milk yield. Milk yield did not show any relationship with suckling behavior or daily gain in calves. Extensive grazing condition did not reduce milk yield of Nellore cows although it determined significant cow weight losses $(80 \mathrm{~kg})$ during the breeding season in the NR system. Daily length of suckling decreased in the NR system as lactation progressed, and was sustained or increased in the intensive systems. Crossbred calves showed higher daily gain and weaning weight than purebred.
\end{abstract}

Key Words: beef cattle, milk yield, suckling behavior

\section{Introdução}

A produção de leite das vacas é uma característica importante na pecuária de corte, uma vez que grande parte dos nutrientes ingeridos pelos bezerros nos primeiros meses de vida provém do leite materno. Várias pesquisas demonstram que o leite materno é responsável por uma porção significativa (de 20 a $25 \%$ ) da variação no peso à desmama e no ganho de peso do nascimento à desmama (ALENCAR, 1989; ALBUQUERQUE et al., 1993).

A quantidade de leite produzido por uma vaca de corte varia em função do seu genótipo e do genótipo do bezerro (DAY et al., 1987). O nível de alimenta- ção também influencia a produção de leite e a expressão do pico de lactação (JENKINS e FERREL, 1992). Maiores produções de leite estão associadas com maiores consumos e ganhos de peso dos bezerros (FISS e WILTON, 1993). Porem, quando as fontes de alimentação são limitadas, as reservas corporais podem ser utilizadas para cobrir requerimentos nutricionais (JENKINS e FERREL, 1992). Vacas alimentadas com maiores níveis de energia na dieta produzem mais leite do que vacas tratadas com baixa energia na dieta (SINCLAIR et al., 1998).

QUADROS e LOBATO (1997) avaliaram a produção de leite de vacas Hereford puras e cruzadas com Charolês, Nelore e Normando em taxas de

\footnotetext{
1 Trabalho financiado pela FAPESP - Parte de Dissertação de Mestrado do primeiro autor (USP/ESALQ).

2 Estudante de Doutorado Convênio PEC/PG - UNESP/Jaboticabal - Prof. Univ. R. O. del Uruguay. E.mail: acespasa@fcav.unesp.br

3 Professor Titular Depto. de Produção Animal ESALQ/USP - Bolsista CNPq. E.mail: iupacker@carpa.ciagri.usp.br

4 Pesquisador Embrapa Pecuária Sudeste - CPPSE - Bolsista CNPq. E.mail: mauricio@cppse.embrapa.br
} 
lotação de 0,6 e $0,8 \mathrm{EV} / \mathrm{ha}$, obtendo diferença significativa entre lotações para produção de leite, produzindo 6,39 e $5,52 \mathrm{~kg} /$ dia. Os autores observaram efeito da interação lotação $\mathrm{x}$ raça, destacando-se a produção de leite das vacas cruzadas, quando em lotação $0,6 \mathrm{EV} / \mathrm{ha}$, indicando que o maior potencial de produção leiteira pode se expressar quando a condição nutricional foi favorável.

Os resultados de SINCLAIR et al. (1998) sugerem que a maioria das raças produtoras de carne mobiliza grandes quantidades de reservas corporais para sustentar a produção de leite. Conseqüentemente, as vacas com altos potenciais de crescimento e relativamente baixos potenciais para produção de leite priorizam o crescimento às expensas da produção de leite, enquanto aquelas com altos potenciais de produção de leite terão suficiente energia para produção de leite e crescimento, sempre que seus requerimentos de mantença sejam relativamente baixos.

Aparentemente, existe um padrão geral de declínio na produção de leite durante a lactação que seria dependente do regime alimentar (MONDRAGON et al., 1983). Nas raças Canchim e Nelore, ALENCAR et al. (1988) observaram queda linear da produção de leite com a progressão da lactação.

O ganho de peso pré-desmama é fortemente influenciado pela produção de leite e a habilidade de amamentação das vacas (YOKOI et al., 1997; MONDRAGON et al., 1983). Alguns resultados indicam que o potencial de crescimento do bezerro não influencia a produção de leite das vacas. $\mathrm{O}$ alto potencial de crescimento dos bezerros pode ser compensado com maior consumo de forragem para satisfazer as demandas nutricionais do crescimento extra, quando comparado com bezerros de menor potencial de crescimento (GRINGS et al., 1996).

Alguns trabalhos têm apresentado relações entre a produção de leite e o comportamento de amamentação. Influências genéticas e ambientais podem afetar o comportamento de amamentação dos bezerros e a produção de leite das vacas (DAY et al., 1987). A raça do bezerro pode influenciar o comportamento de amamentação. DAS et al. (2000) observaram que o tempo diário de amamentação varia com a raça, sendo significativamente superior para bezerros zebuínos (11,8 minutos) comparados com bezerros cruzados (9,4 minutos). Os bezerros zebuínos apresentaram duração de cada mamada significativamente superior aos bezerros cruzados (2,8 vs. 2,3 minutos), assim como maiores freqüências de mamadas por dia (2,8 vs. 2,2, respectivamente). Altas produções de leite e altos pesos de bezerros têm sido associados a menores números de mamadas (ODDE et al., 1985).

O tempo total de amamentação por dia decresce com o aumento da idade dos bezerros de diferentes grupos genéticos (DAS et al., 2000).

Segundo RIBEIRO et al. (1991), vacas amamentando bezerros mestiços produziram $29 \%$ mais leite comparativamente àquelas que amamentaram bezerros puros. ALENCAR et al. (1995) encontraram efeitos significativos do grupo genético do bezerro sobre o número e a duração das mamadas, para bezerros das raças Canchim e "meio sangue" Canchim-Nelore.

Em experimentos realizados com várias raças européias e cruzadas européias com zebuínas, o número total de mamadas declina com o avanço da idade do bezerro (REINHARDT e REINHARDT, 1981), mas a duração de cada mamada não muda (DAY et al., 1987).

Houve tendência de maiores produções de leite em vacas amamentando bezerros machos do que fêmeas, apesar de ser significativa só para a produção de leite diária aos 210 dias de lactação (ALENCAR et al., 1988). Vacas da raça Canchim amamentando fêmeas produziram $12 \%$ mais leite total do que aquelas com machos, enquanto nas vacas da raça Nelore ocorreu o inverso, ou seja, as vacas que amamentaram machos produziram 19\% mais leite total (CRUZ et al., 1997). Outras pesquisas não encontraram diferenças entre sexos para o comportamento de amamentação (REINHARDT e REINHARDT, 1981).

O objetivo deste trabalho consistiu em avaliar a produção de leite de vacas Nelore amamentando bezerros puros (em sistemas de manejo extensivo e intensivo) ou cruzados (em sistemas de manejo intensivo), bem como o crescimento e o comportamento de amamentação dos bezerros durante a fase pré-desmama.

\section{Material e Métodos}

O trabalho faz parte do Projeto "Estratégias de cruzamentos, práticas de manejo e biotécnicas para intensificação sustentada da produção de carne bovina", Subprojeto "Avaliação de diferentes sistemas de cruzamento entre raças bovinas de corte" da Embrapa Pecuária do Sudeste. O projeto visa comparar, sob diversos aspectos, os seguintes sistemas de produção (SIST): NR-Nelore Referência, sob manejo extensivo; NI-Nelore, sob manejo intensivo; e três cruzamentos $\mathrm{CN}$ - Canchim x Nelore, AN - Angus x Nelore e 
704 Rev. bras. zootec.

SN - Simental x Nelore, sob manejo intensivo.

O sistema NR simulou condições de manejo tradicional sob pastejo em Brachiaria decumbens com taxa de lotação de $1 \mathrm{UA} /$ ha. Os sistemas NI, CN, AN e SN foram manejados em pastejo de Brachiaria brizantha cv. Marandu, com taxas de lotação de $5 \mathrm{UA} / \mathrm{ha}$, incluindo suplementação de vacas e bezerros durante a época da seca.

Em 12 vacas de cada sistema de produção (NR, NI, CN, AN e SN), foram avaliadas a produção de leite aos 60, 120 e 180 dias após o parto $\left(\mathrm{PL}_{60}, \mathrm{PL}_{120}\right.$ e $\mathrm{PL}_{180}$ ), pela técnica da pesagem do bezerro antes e após a mamada, e a variação de peso durante a estação de monta (VP).

Nos bezerros (12 em cada sistema de produção), foram calculados os ganhos de peso diário entre $0 \mathrm{e}$ 60 dias $\left(\mathrm{GD}_{0-60}\right), 60$ e 120 dias $\left(\mathrm{GD}_{60-120}\right)$ e 120 e 180 dias após o parto $\left(\mathrm{GD}_{120-180}\right)$ (momentos $1,2 \mathrm{e}$ 3 , respectivamente), e o peso à desmama (PD).

No dia seguinte a cada determinação da produção de leite (PL), foi observado o comportamento de amamentação dos bezerros pelo número (NM) e duração de cada mamada (MinM), das 6 às $18 \mathrm{~h}$. O tempo diário de amamentação (MinD) foi calculado pelo produto entre o número e a duração das mamadas.

A produção de leite e o comportamento de amamentação (NM, MinM e MinD), considerados como medidas repetidas no tempo, foram analisados conforme o seguinte modelo misto:

$$
\begin{aligned}
\mathrm{Y}_{\mathrm{ijklm}} & =\mu+\mathrm{SIST}_{\mathrm{i}}+\mathrm{SEXO}_{\mathrm{j}}+\mathrm{b}_{1} \mathrm{IV}_{\mathrm{k}}+\mathrm{Vaca}(\mathrm{SIST})_{1(\mathrm{i})} \\
& +\mathrm{MOM}_{\mathrm{m}}+\mathrm{SIST}^{* \operatorname{MOM}_{(\mathrm{im})}}+\mathrm{e}_{\mathrm{ijklm}}
\end{aligned}
$$

em que $\mu$ é média; $Y_{\mathrm{ijklm}}$, produção diária de leite (Kg), NM, MinM e MinD; SIST ${ }_{\mathrm{i}}$, efeito fixo do sistema de produção (NR, NI, CN, $\mathrm{AN}$ e $\mathrm{SN}$ ); $\mathrm{SEXO}_{j}$, efeito fixo do sexo do bezerro; $\mathrm{b}_{1} \mathrm{IV}_{\mathrm{k}}$, covariável idade da vaca; VACA(SIST) $)_{\mathrm{m}(\mathrm{i})}$, efeito aleatório da vaca dentro do sistema de produção; $\mathrm{MOM}_{\mathrm{m}}$, efeito fixo do momento da lactação $(60,120$ e 180 dias após o parto); SIST*MOM $(\mathrm{im})$, interação entre sistema de produção e momento da lactação; $\mathrm{e}_{\mathrm{ijklm}}$, resíduo.

A persistência da produção de leite foi estudada por meio da produção final (180 dias) ajustada pela produção inicial (60 dias após o parto), mediante modelo:

$\mathrm{PL} 180_{\mathrm{ijkl}}=\mu+\mathrm{SIST}_{\mathrm{i}}+\mathrm{SEXO}_{\mathrm{j}}+\mathrm{b}_{1} \mathrm{IV}_{\mathrm{k}}+$ $\mathrm{b}_{2} \mathrm{PL}_{1}+\mathrm{VACA}(\mathrm{SIST})_{\mathrm{m}(\mathrm{i})} \mathrm{SIST}_{1} \mathrm{PL} \mathrm{O}_{(\mathrm{il})}+\mathrm{e}_{\mathrm{ijkl}}$

As relações entre as variáveis ganho de peso, produção de leite e comportamento de amamentação foram estudadas por meio de análises de regressão e correlação (SAS, 1997).

Os ganhos de peso em cada momento foram analisados por meio de modelo similar ao descrito para produção de leite, contendo também as produções de leite como covariáveis. Os ganhos de peso dos bezerros dos diferentes sistemas de produção foram comparados mediante contrastes ortogonais: Puros vs. Cruzados; NR vs. NI; Britânicos (AN) vs. Continentais (CN, SN); e Canchim vs. Simental.

As análises de variância foram realizadas pelo método REML, por meio do procedimento MIXED do programa SAS (1997), usando estimativas de covariância do tipo $\mathbf{U N}$, mediante modelo selecionado pelo critério de Akaike, conforme sugerido para medidas repetidas (LEITTELL et al., 1996). Sempre que necessárias, foram estimadas as médias ajustadas e comparadas entre si pelo teste $t$.

\section{Resultados e Discussão}

A produção de leite foi influenciada significativamente pelo $\mathrm{MOM}$ da lactação $(\mathrm{P}<0,001)$ e pela interação SIST*MOM $(\mathrm{P}<0,02)$, enquanto o tempo diário de amamentação foi influenciado $(\mathrm{P}<0,02)$ pela interação SIST*MOM.

Na Tabela 1 são apresentadas as médias ajustadas da produção de leite para cada um dos cinco sistemas de produção estudados e, na Figura 1, as médias são representadas graficamente.

Tabela 1 - Produção de leite (kg/dia) nos cinco sistemas de produção em três momentos da lactação

Table 1 - Milkyield (kg/day) in five production systems in three lactation moments

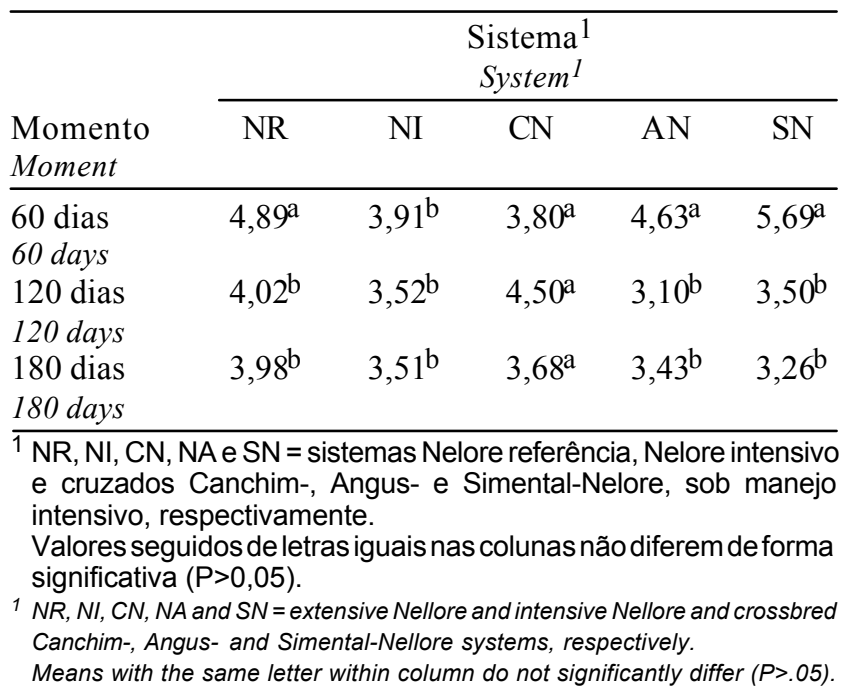


A PL decresce de forma apreciável nos sistemas $\mathrm{NR}, \mathrm{AN}$ e SN, com o avanço da lactação, enquanto para os sistemas NI e CN permanece constante. A diminuição da produção de leite com o avanço da lactação concorda com os resultados encontrados por ALENCAR et al. (1988), que observaram queda da produção de leite de modo linear e significativo.

Ao contrário do observado por QUADROS e LOBATO (1997), a produção de leite não apresentou valores superiores nos sistemas de produção com manejos intensivos.

Para a persistência da produção de leite $\left(\mathrm{PL}_{180}\right)$, houve efeitos significativos do sistema de produção $(\mathrm{P}<0,02)$ e da interação SIST*PL $60(\mathrm{P}<0,04)$. Somente no sistema NR foi encontrado efeito significativo da produção de leite aos 60 dias após o parto sobre a produção de leite aos 180 dias $(\mathrm{P}<0,008)$. Estes resultados acompanham as tendências publicadas por SINCLAIR et al. (1998), indicando que a vaca Nelore priorizará a produção de leite ainda em condições de alimentação adversas.

Conforme publicado por JENKINS e FERREL (1992), provavelmente a produção de leite do sistema NR tenha sido mantida mediante mobilização das reservas corporais, o que pode ser comprovado pela perda de peso durante a lactação exibida pelas vacas do sistema NR em comparação com os sistemas intensivos (80 vs. 20, 8, 11 e $11 \mathrm{~kg}$ para NR, NI, CN, $\mathrm{AN}$ e $\mathrm{SN}$, respectivamente).

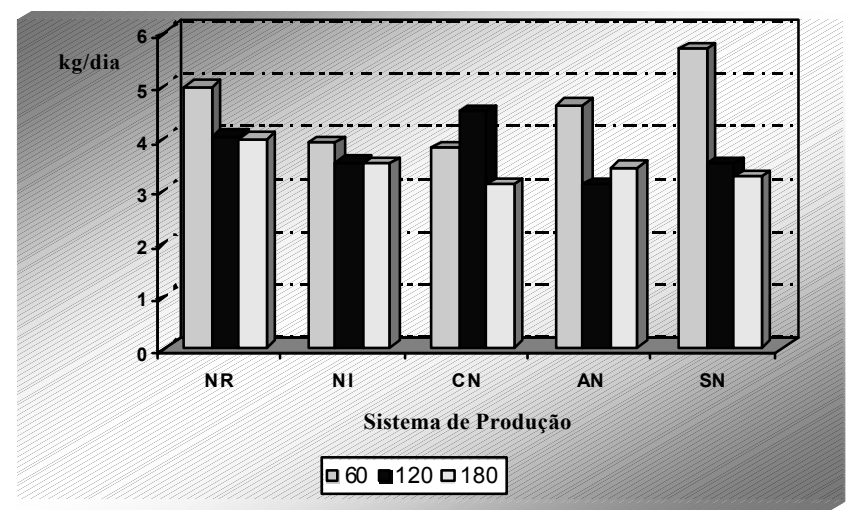

Figura 1 - Produção de leite durante a lactação em cinco sistemas de produção (NR, NI, CN, NA e SN = sistemas Nelore referência, Nelore intensivo e cruzados Canchim, Angus e Simental - Nelore, sob manejo intensivo, respectivamente.).

Figure 1 - Milk yield on lactation in five production systems (NR, $\mathrm{NI}, \mathrm{CN}, N A$ and $S N=$ extensive Nellore and intensive Nellore and crossbred Canchim, Angus, and Simental - Nellore systems, respectively).
A produção total de leite foi comparada pelos mesmos contrastes ortogonais que o comportamento de amamentação. Não foram encontradas diferenças significativas entre os grupos comparados, o que permite concluir que o tipo de manejo (intensivo e extensivo) e o grupo genético do bezerros não influenciaram a produção de leite das vacas Nelore.

Não foi observado efeito do sexo do bezerro para nenhuma das variáveis estudadas (PL, NM, MinM, MinD), ao contrário dos resultados encontrados por ALENCAR et al. (1988) e CRUZ et al. (1997).

Contrariamente ao observado por REINHARDT e REINHARDT (1981), o número de mamadas por dia não apresentou variação durante a lactação, permanecendo com a média de duas mamadas por dia. Este comportamento pode ser explicado pela prática da suplementação de vacas e bezerros, interferindo no comportamento normal de pastejo e na amamentação.

Em contraposição aos resultados de DAY et al. (1987), foi observada tendência $(\mathrm{P}<0,01)$ de declínio no tempo total de duração de cada mamada com o avanço da lactação, acompanhando a redução na produção de leite dos 60 aos 180 dias após o parto (4,6 vs. $3,5 \mathrm{~kg} / \mathrm{dia}$, respectivamente, $\mathrm{P}<0,0001$ ).

$\mathrm{Na}$ Tabela 2 são apresentados os tempos de duração de cada mamada para os cinco sistemas de produção estudados. De modo similar ao publicado por RIBEIRO et al. (1991) e ALENCAR et al. (1995), o sistema de produção (puros e cruzados) teve efeito significativo sobre o tempo de duração de cada mamada $(\mathrm{P}<0,05)$. Os bezerros $\mathrm{SN}$ apresentaram maior tempo de duração de cada mamada, seguidos dos bezerros do sistema Nelore Intensivo. Observaram-se também, apesar de não significativo, menores durações das mamadas nos bezerros do sistema extensivo, quando comparados aos intensivos.

$\mathrm{O}$ tempo diário de amamentação dos bezerros também foi influenciado significativamente pela interação SIST*MOM $(\mathrm{P}<0,04)$. Na Tabela 3 são apresentadas as médias ajustadas do tempo diário de amamentação dos cinco sistemas de produção, avaliados nos três momentos da lactação e, na Figura 2, as médias são representadas graficamente.

O sistema NR mostrou tendência diferente à dos outros quatro sistemas de produção avaliados. Nesse sistema, o tempo diário de amamentação decresceu com o avanço da lactação (19,98 vs. 9,28 minutos/dia, $\mathrm{P}<0,003)$. Estas observações concordam com os resultados publicados por DAS et al. (2000), em que o tempo total de amamentação por dia decresce de forma significativa com o avanço da idade dos bezer- 
706 Rev. bras. zootec.

Tabela 2 - Tempo de duração de cada mamada (minutos) para cinco sistemas de produção

Table 2 - Time of sukling (minutes) for five production systems

Sistema de produção ${ }^{1}$

Production system ${ }^{1}$

Duração média das mamadas (minutos)

Lenght of suckles (minutes)

\begin{tabular}{ll}
\hline NR & $6,33^{\mathrm{b}}$ \\
NI & $7,09^{\mathrm{ab}}$ \\
CN & $6,52^{\mathrm{b}}$ \\
AN & $6,82^{\mathrm{b}}$ \\
SN & $7,96^{\mathrm{a}}$
\end{tabular}

${ }^{1} \mathrm{NR}, \mathrm{NI}, \mathrm{CN}, \mathrm{NA}$ e SN = sistemas Nelore referência, Nelore intensivo e cruzados Canchim-, Angus- e Simental-Nelore, sob manejo intensivo, respectivamente.

Valores seguidos de letras iguais não diferem de forma significativa $(\mathrm{P}>0,05)$.

$1 \mathrm{NR}, \mathrm{NI}, \mathrm{CN}, \mathrm{NA}$ and $\mathrm{SN}=$ extensive Nellore and intensive Nellore and crossbred Canchim-, Angus-, and Simental-Nellore systems, respectively.

Means with the same letter within column do not significantly differ $(P<.05)$.

ros. Provavelmente, as condições limitantes de alimentação, nas quais os animais foram manejados (taxa de lotação de 1,0 UA/ha), influenciaram o número e a duração das mamadas dos bezerros, em favor de um aumento do tempo diário de pastejo de vacas e bezerros.

A redução observada no tempo diário de amamentação do sistema NR esteve acompanhada por redução significativa da produção diária de leite das vacas (4,96 vs. $3,98 \mathrm{~kg} / \mathrm{dia}$, Tabela 1$)$.

Os sistemas de produção com manejos intensivos não apresentaram mudanças significativas, com exceção dos sistemas $\mathrm{CN}$ e SN, os quais aumentaram os tempos diários de amamentação (13,51 vs. 17,11 minutos/dia e 13,13 vs. 20,05 minutos/dia, $\mathrm{P}<0,05$ ), com o avançar da lactação.

Ao contrário do esperado, observaram-se tempos diários de amamentação (9,28 a 20,05 minutos/dia) com médias de magnitudes superiores às publicadas por DAS et al. (2000) para bezerros cruzados e zebuínos (9,4 a 11,8 minutos/mamada, respectivamente).

Conforme observado para a produção de leite, também não foram registradas diferenças entre sexos em relação aos minutos de amamentação por dia, concordando com o publicado por REINHARDT e REINHARDT (1981).

As análises de regressão e correlação realizadas não evidenciaram relação entre número e duração das mamadas e produção de leite $(\mathrm{P}>0,9)$, diferentemente do observado por ODDE et al. (1985), em que as maiores produções de leite estiveram associadas com menores números de mamadas por dia.
Tabela 3 - Tempo diário de amamentação (minutos/dia) nos cinco sistemas de produção avaliados

Table 3 - Daily suckling lenght in five production systems

\begin{tabular}{lccccc}
\hline & \multicolumn{5}{c}{$\begin{array}{c}\text { Sistema }^{1} \\
\text { System }\end{array}$} \\
\cline { 2 - 6 } $\begin{array}{l}\text { Momento } \\
\text { Moment }\end{array}$ & NR & NI & CN & AN & SN \\
\hline 60 dias & $19,98^{\mathrm{a}}$ & $14,33^{\mathrm{a}}$ & $13,51^{\mathrm{a}}$ & $13,75^{\mathrm{a}}$ & $13,13^{\mathrm{b}}$ \\
60 days \\
$\begin{array}{l}120 \text { dias } \\
120 \text { days }\end{array}$ & $14,49^{\mathrm{ab}}$ & $11,99^{\mathrm{a}}$ & $17,91^{\mathrm{a}}$ & $13,51^{\mathrm{a}}$ & $13,56^{\mathrm{ab}}$ \\
180 dias & $9,28 \mathrm{~b}$ & $12,65^{\mathrm{a}}$ & $17,11^{\mathrm{a}}$ & $14,04^{\mathrm{a}}$ & $20,05^{\mathrm{a}}$ \\
180 days & & & & &
\end{tabular}

days

${ }^{1} \mathrm{NR}, \mathrm{NI}, \mathrm{CN}, \mathrm{NA} \mathrm{e} \mathrm{SN} \mathrm{=} \mathrm{sistemas} \mathrm{Nelore} \mathrm{referência,} \mathrm{Nelore} \mathrm{intensivo}$ e cruzados Canchim-, Angus- e Simental-Nelore, sob manejo intensivo, respectivamente.

Valores seguidos de letras iguais nas colunas não diferem de forma significativa $(P>0,05)$.

${ }^{1} \mathrm{NR}, \mathrm{NI}, \mathrm{CN}, \mathrm{NA}$ and $\mathrm{SN}=$ extensive Nellore and intensive Nellore and crossbred Canchim- , Angus- and Simental-Nellore systems, respectively.

Means with the same letter within column do not significantly differ $(P>05)$.

Os ganhos em peso dos bezerros (GD0-60, GD60120, GD120-180) não estiveram relacionados nem com a produção de leite nem com a atividade de amamentação dos bezerros. Estes resultados não permitem observar a forte influência da produção de leite e a habilidade de amamentação sobre o ganho de peso pré-desmama publicada em inúmeros trabalhos (MONDRAGON et al., 1983; YOKOI et al., 1997), provavelmente por causa do momento no qual foram começadas as determinaçöes (60 dias após o nascimento). Não foi observada a dependência exclusiva do leite materno nas primeiras etapas da lactação conforme o encontrado por ALENCAR (1989), uma vez que as maiores produções de leite (Tabela 1 ) nem sempre foram acompanhadas dos maiores ganhos de peso dos bezerros.

Na Tabela 4 são apresentadas as médias ajustadas para os ganhos em peso e os pesos à desmama dos bezerros dos diferentes sistemas estudados.

$\mathrm{O}$ grupo NR apresentou os menores ganhos em peso durante toda fase pré-desmama $(0,72 ; 0,57 \mathrm{e}$ $0,45 \mathrm{~kg} /$ dia nos períodos 1,2 e 3, respectivamente), enquanto os grupos $\mathrm{AN} \mathrm{e} \mathrm{SN} \mathrm{apresentaram} \mathrm{os} \mathrm{maiores}$ ganhos de peso para os mesmos períodos $(0,84 ; 0,84 \mathrm{e}$ 0,85 ; e 0,$85 ; 0,80$ e 0,83 , respectivamente).

Os menores ganhos do sistema NR podem ser explicados pelas condições diferentes de alimentação às quais foram submetidos. É evidente que a pastagem oferecida aos sistemas NI, CN, AN e SN, assim como a suplementação, favoreceu maiores ganhos diários. Isto é evidenciado pelo ganho de peso 


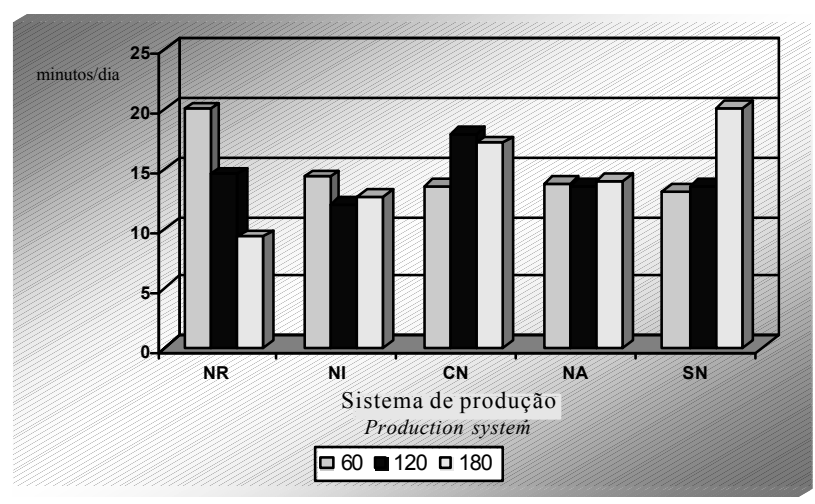

Figura 2 - Tempo diário de amamentação (minutos/dia)aos 60,120 e 180 dias após o parto (NR, NI, CN, NA e $\mathrm{SN}=$ sistemas Nelore referência, Nelore intensivo e cruzados Canchim-, Angus- e Simental-Nelore, sob manejo intensivo, respectivamente).

Figure 2 - Daily suckling lenght (minutes/day) in 60, 120 and 180 days after calving (NR, NI, CN, NA and SN = extensive Nellore and intensive Nellore and crossbred Canchim-, Angus-, and Simental-Nellore systems, respectively.)

Tabela 4 - Ganhos diários pré-desmama e pesos à desmama de bezerros puros e cruzados

Table 4 - Daily gain pre weaning and pure breed and cross calf weaning weight

\begin{tabular}{lcccc}
\hline $\begin{array}{l}\text { Sistema } \\
\text { Production } \\
\text { system }\end{array}$ & $\begin{array}{c}\text { GD0-60 } \\
(\mathrm{kg} / \mathrm{dia})\end{array}$ & $\begin{array}{c}\text { GD60-120 } \\
(\mathrm{kg} / \mathrm{dia})\end{array}$ & $\begin{array}{c}\text { GD120-180 } \\
(\mathrm{kg} / \mathrm{dia})\end{array}$ & $\begin{array}{c}\mathrm{PD} \\
(\mathrm{kg})\end{array}$ \\
\hline $\mathrm{NR}$ & $0,72^{\mathrm{b}}$ & $0,57^{\mathrm{c}}$ & $0,45^{\mathrm{c}}$ & $136^{\mathrm{c}}$ \\
$\mathrm{NI}$ & $0,76^{\mathrm{ab}}$ & $0,70^{\mathrm{b}}$ & $0,65^{\mathrm{b}}$ & $161^{\mathrm{b}}$ \\
$\mathrm{CN}$ & $0,83^{\mathrm{ab}}$ & $0,75^{\mathrm{ab}}$ & $0,80^{\mathrm{ab}}$ & $172^{\mathrm{b}}$ \\
$\mathrm{AN}$ & $0,84^{\mathrm{a}}$ & $0,84^{\mathrm{a}}$ & $0,85^{\mathrm{a}}$ & $189^{\mathrm{a}}$ \\
$\mathrm{SN}$ & $0,85^{\mathrm{a}}$ & $0,80^{\mathrm{ab}}$ & $0,83^{\mathrm{a}}$ & $188^{\mathrm{a}}$ \\
\hline
\end{tabular}

${ }^{1} \mathrm{NR}, \mathrm{NI}, \mathrm{CN}, \mathrm{NA} \mathrm{e} \mathrm{SN}=$ sistemas Nelore referência, Nelore intensivo e cruzados Canchim-, Angus- e Simental-Nelore, sob manejo intensivo, respectivamente.

Valores seguidos de letras iguais nas colunas não diferem de forma significativa $(P>0,05)$.

$1 \mathrm{NR}, \mathrm{NI}, \mathrm{CN}, \mathrm{NA}$ and $\mathrm{SN}=$ extensive Nellore and intensive Nellore and crossbred Canchim - , Angus -, and Simental-Nellore systems, respectively.

Means with same letter within column do not significantly differ $(P>.05)$

realizado pelos bezerros Nelore, quando manejados em condições intensivas. No entanto, também é evidenciada superioridade dos bezerros cruzados.

Os grupos NI e CN mostraram desempenhos intermediários $(0,76 ; 0,70$ e 0,65 ; e 0,$83 ; 0,75$ e 0,80 , respectivamente). Os ganhos de peso superiores dos bezerros cruzados, em relação aos dos grupos puros, estão de acordo com o publicado por ALENCAR (1997).

O sistema de manejo tradicional seguramente comprometeu o desenvolvimento normal dos bezer- ros e vacas, observando-se perdas de peso comparativamente superiores nas vacas manejadas em condições extensivas ( $80 \mathrm{~kg}$ vs. 20, 13, 11 e 11 para NR, $\mathrm{NI}, \mathrm{CN}, \mathrm{AN}$ e SN respectivamente), assim como pesos à desmama significativamente menores (136 vs. $161,172,189$ e 188 , para NI, CN, AN e SN respectivamente).

Os diferentes sistemas de produção foram comparados por meio de contrastes ortogonais, os quais demonstraram nítida superioridade dos sistemas de manejo intensivo em relação ao sistema NR, durante toda a fase pré desmama $(\mathrm{P}<0,0001)$.

Os pesos à desmama dos bezerros cruzados com raças européias superaram de forma significativa os pesos dos puros, independentemente do plano de alimentação (136 e $161 \mathrm{~kg}$ para NR, NI vs. 189 e $188 \mathrm{~kg}$ para $\mathrm{AN} \mathrm{e} \mathrm{SN,} \mathrm{respectivamente),} \mathrm{ao} \mathrm{passo} \mathrm{que} \mathrm{o} \mathrm{desempenho}$ do sistema $\mathrm{CN}$ foi intermediário entre os anteriores. $\mathrm{O}$ crescimento dos bezerros do grupo NI superou o dos NR, porém foi inferior aos cruzados, demonstrando que a melhora no ambiente não foi suficiente para igualar o desempenho dos bezerros cruzados.

De acordo com o publicado por GRINGS et al. (1996), os maiores ganhos de peso obtidos pelos bezerros cruzados possivelmente foram acompanhados de maiores requerimentos nutricionais. Provavelmente, estes requerimentos tenham sido compensados com maiores ingestões de forragem e suplemento, uma vez que as vacas que amamentaram bezerros cruzados não foram sempre as que produziram mais leite.

\section{Conclusões}

A produção de leite varia com o momento da lactação e a interação sistema de produção x momento da lactação.

A produção de leite durante a fase pré-desmama não apresentou relação direta com o comportamento de amamentação, nem o ganho de peso dos bezerros dos diferentes sistemas de produção.

Condições deficientes de alimentação não resultaram em menores produções de leite de vacas Nelore, mas sim em acentuadas perdas de peso.

O tempo diário de amamentação apresentou queda significativa no sistema extensivo durante a lactação, enquanto os sistemas intensivos não mudaram ou aumentaram os minutos de amamentação por dia.

Para as condições nas quais o experimento foi desenvolvido, os bezerros cruzados apresentaram os melhores desempenhos durante a fase pré-desmama, em comparação com os puros. 


\section{Agradecimento}

Ao convênio PEC/PG e à ESALQ, pela oportunidade oferecida para a realização do Mestrado.

Á Empresa Brasileira de Pesquisa Agropecuária (EMBARAP-CPPSE) e seu pessoal, pela oportunidade de trabalhar nas suas dependências, assim como pela colaboração permanente durante a realização do trabalho.

A Universidad de la República Oriental del Uruguay (Fac. de Agronomía e CSIC).

\section{Referências Bibliográficas}

ALENCAR, M.M., RUZZA, F.J., PORTO, E.J.S. 1988. Desempenho produtivo de fêmeas das raças Canchim e Nelore. III. produção de leite Rev. Soc.Bras.Zootec., 17(4):317-328.

ALENCAR, M.M. 1989. Relação entre produção de leite da vaca e desempenho do bezerro nas raças Canchim e Nelore. R. Soc. Bras. Zootec., 18(2):146-156.

ALENCAR, M.M., CRUZ, G.M., TULLIO, R.R. et al. 1995. Características da amamentação de bezerros da raça Canchim e cruzados Canchim x Nelore. R. Soc. Bras. Zootec., 24(5):706-714.

ALENCAR, M.M. Los cruzamentos para la producción de carne bovina. In: CONGRESO INTERNACIONAL DE TRANSFERENCIA DE TECNOLOGÍA AGROPECUARIA, 1997, Asunción. Anais... Asunción: CEA, 1997. p.111-122.

ALBUQUERQUE, L.G., ELER, J.P., COSTA, M.R.P. et al. 1993. Produção de Leite e desempenho do Bezerro na fase de Aleitamento em três raças bovinas de corte. R. Soc. Bras. Zootec., 22(5):745-754.

CRUZ, G.M., ALENCAR, M.M., TULLIO, R.R. 1997. Produção e composição do leite de vacas das raças Canchim e Nelore. R. Soc. Bras. Zootec, 26(5):887-893.

DAS, S.M., REDBO, I., WIKTORSSON, H. 2000. Effect of age of calf on suckling behaviour and other behavioural activities of Zebu and crossbred calves during restricted suckling periods. Appl. Anim. Behav. Sci., 67(1-2):47-57.

DAY, M.L., IMAKAWA, K., CLUTTER, A.C. et al. 1987. Suckling behavior of calves with dams varying in milk production. J. Anim. Sci., 65:1207-1212.

FISS, C.F., WILTON, J.W. 1993. Contribution of breed, cow weight, and milk yield to the preweanning, feedlot and carcass traits of calves in three beef breeding systems. J. Anim. Sci., $71: 2874-2884$.
GRINGS, E.E., SHORT, R.E., McNEIL, M.D. et al. 1996. Efficiency of production in cattle of two growth potencials on northorn great plains rangelands during spring-summer grazing. J. Anim. Sci., 74:2317-2326.

JENKINS, T.G., FERREL, C.L. 1992. Lactation characteristics of nine breeds of cattle fed various quantities of dietary energy.J. Anim. Sci., 70:1652-1660.

LEITTELL, R.C., MILLIKEN, G.A., STROUP, W. W. et al. 1996. System for Mixed Models. Cary: SAS Institute, $633 \mathrm{p}$

MONDRAGON, I., WILTON, J.W., ALLEN, O.B. et al. 1983. Stage of lactation effects, repeteabilities and influences on weaning weights of yield and composition of milk in beef cattle. Can. J. Anim. Sci., 63:751-761.

ODDE, K.G., KIRACOFE, G.H., SCHALLES, R.R. 1985. Suckling behavior in range beef calves. J. Anim. Sci., 61(2):307-309.

QUADROS, S.A.F., LOBATO, J.F.P. 1997. Efeitos da lotação animal na produção de leite de vacas de corte primíparas e no desenvolvimento de seus bezerros. R. Soc. Bras. Zootec., 26(1):27-33.

REINHARDT, V., REINHARDT, A. 1981. Natural suckling performance and age of weaning in zebu cattle (Bos indicus). J. Agric. Sci., 96:309-312.

RIBEIRO, E.L.A., RESTLE, J., PIRES, C.C. 1991. Produção e composição do leite em vacas Charolês e Aberdeen Angus amamentando terneiros puros ou mestiços. Pesq. Agropec. Bras., 26(8):1267-1273.

SAS. Statistical Analysis System User's Guide: Stat, Version 6.12 Cary:SAS Institute. 1997.

SINCLAIR, K.D., YILDIZ, S., QUINTANS, G. et al. 1998. Annual energy intake and the performance of beef cows differing in body size and milk potential. Anim. Sci., 66:643-655.

YOKOI, N., MORIYA, K., SASAKI, Y. 1997. A mesure for predicting genetic merit for milking and nursing ability in beef cattle. Anim. Sci., 65:39-43.

Recebido em: $26 / 07 / 00$

Aceito em: 24/01/01 